\title{
Guest editor's preface
}

The accuracy and the level of quality that characterize estimates derived from surveys are inseparably linked to the underlying survey design and its success in satisfying response rate targets, precision requirements, and controlling remaining sources of survey error. The papers that are published in this special issue of the Journal of Economic and Social Measurement focus on statistical and methodological investigations that provide major insights regarding the impact of survey nonresponse and other sources of survey error on resultant estimates. Particular attention is given to the topics of bias and precision, the dual components of mean square error that affect the overall quality of survey estimates. The statistical and methodological studies that are highlighted in this volume are further connected by their substantive focus on the measurement of health care parameters at the national level that summarize the population's health care utilization, expenditures, insurance coverage, sources of payment, access to care and quality of health care.

The health care survey that is highlighted in these analytical evaluations is the Medical Expenditure Panel Survey (MEPS). The MEPS was designed to produce national and regional annual estimates of the health care utilization, expenditures, sources of payment and insurance coverage of the US civilian non-institutionalized population. The scope and depth of this data collection effort reflects the needs of government agencies, legislative bodies, and health professionals for the comprehensive national estimates needed in the formulation and analysis of national health policies. The survey is sponsored by the Agency for Healthcare Research and Quality (AHRQ) with co-sponsorship from the National Center for Health Statistics. Analytical enhancements have already been realized in the MEPS as a consequence of design modifications that changed the survey's periodicity from a ten year cycle to an on-going annual national medical expenditure survey, the inclusion of a longitudinal panel covering the health care experience over a two year period, and a design capacity that permits an efficient oversampling of policy relevant population subgroups. Since many of the design features of the MEPS are similar to other national survey efforts both within and outside the field of health, the research findings that are presented in this special volume should serve to inform design improvements for comparable survey efforts.

The level of bias in estimates attributable to nonresponse is a function of the level of nonresponse experienced by the survey and the magnitude of the difference in the parameter estimates that distinguish respondents from nonrespondents. Consequently, the achievement of a high rate of response for a survey will serve to limit the impact of nonresponse bias on resultant survey estimates. To inform the development of effective nonresponse adjustment strategies in panel surveys, the paper 
"Survey attrition considerations in the Medical Expenditure Panel Survey" identifies characteristics that distinguish the survey participants from those that attrited over time and examines the impact of survey attrition on resultant survey estimates of health insurance coverage. Both the data collection and estimation strategies that were implemented to mitigate the impact of nonresponse bias associated with survey attrition in the MEPS should serve as an effective model that warrants replication. This effort is complemented by the paper "An alternative measure of response rate in random-digit-dialing surveys that screen for eligible subpopulations", which provides a foundation to guide the derivation of response rates when there is uncertainty in the eligibility status of a subset of sampled cases.

Survey design efforts are also directed toward achieving additional reductions in other sources of survey error, subject to existing budget constraints. This is most evident in sample design analyses directed towards minimizing costs associated with data collection efforts, subject to fixed precision specifications and requirements with respect to statistical power. The next paper, entitled "The impact of alternative sample allocation schemes on the precision of survey estimates derived from the National Medical Expenditure Panel Survey" evaluates the impact of alternative sampling allocation schemes in a national multistage health care survey on precision and cost in a national multistage health care survey. The discussion of trade-offs between cost and precision in a national survey with a geographically dispersed multi-stage sample design should serve to inform decisions regarding the appropriate level of geographic clustering to consider for national survey efforts.

In the spirit of identifying methodologies that help ensure other sources of survey error are reduced beyond those attributable to nonresponse, sample size and design, the next paper in this volume focuses on the topic of measurement error. More specifically, the paper "Probability matching of medical events" addresses sources of measurement error in household reports of medical utilization and expenses and identifies methodologies to reduce error in match rates between household and medical provider event level data covering the same set of individuals. The medical events reported by the two sources are subject to reporting differences and probability linkage methods are used to determine if pairs of medical events represent the same entities. The study results also serve to inform an evaluation of the level of accuracy inherent in household reports of their medical utilization experience.

The final paper on "Measuring inpatient care use in the United States: A comparison across five federal data sources" complements the other papers by focusing on the benchmarking of estimates across alternative surveys. It serves to provide insights with respect to issues of data comparability and the need to ensure standardization in target populations and underlying units of measurement when conducting the comparisons.

The research findings presented in these manuscripts have significantly contributed to the enhancements and innovations that characterize the current survey design of the ongoing Medical Expenditure Panel Survey. It is the hope of this author that 
some of the statistical and methodological results that have been presented will serve to improve the quality of other related survey efforts.

Steven B. Cohen, Ph.D.

Acting Director, Center for Cost and Financing Studies Director, Division of Statistical Research and Methods

Agency for Healthcare Research and Quality 2101 East Jefferson Street, Suite 500

Rockville, MD 20852

USA 